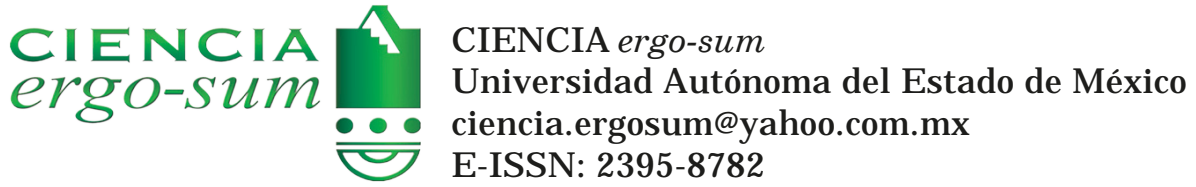

\title{
Descubrimiento de conocimiento en incidencia de tipo de cáncer para pacientes terminales mediante minería de datos
}

Gutiérrez Pérez, Luis Antonio; Gutiérrez Cruz, Doricela; Rico Molina, Ricardo; Rodríguez Páez, Carmen
Liliana; Albarrán Fernández, Yaroslaf Aarón; Soto Rivera, Bernardo; Gutiérrez Cruz, Alma Rebeca;
Duran López, Víctor Manuel
Descubrimiento de conocimiento en incidencia de tipo de cáncer para pacientes terminal es mediante
minería de datos
CIENCIA ergo-sum, vol. 28, núm. 1, marzo-junio $2021 \mid$ e111
Universidad Autónoma del Estado de México, México

Gutiérrez Pérez, L. A.; Gutiérrez Cruz, D.; Rico Molina, R.; Rodríguez Páez, C. L.; Albarrán Fernández, Y. A.; Soto Rivera, B.; Gutiérrez Cruz, A. R. y Duran López, V. M. (2021). Descubrimi ento de conocimiento en incidencia de tipo de cáncer para pacientes termi nales mediante minería de datos. CI E NCIA ergo-sum, 28(1). https://doi.org/10.30878/ces.v28n1a5 


\section{Descubrimiento de conocimiento en incidencia de tipo de cáncer para pacientes terminales mediante minería de datos}

\section{Discovery of knowledge on incidence of cancer type for terminal patients through data mining}

Luis Antonio Gutiérrez Pérez

Universidad Autónoma del Estado de México, México

luis_uaem@yahoo.com

Recepción: 06 de diciembre de 2018

(D) http://orcid.org/0000-0002-9636-4639

Aprobación: 19 de septiembre de 2019

Doricela Gutiérrez Cruz

Universidad Autónoma del Estado de México, México

dgutierrezcr@uaemex.mx

(1) http://orcid.org/0000-0003-2843-3273

Ricardo Rico Molina

Universidad Autónoma del Estado de México, México

rricom@uaemex.mx

(D) http://orcid.org/0000-0001-9586-8758

Carmen Liliana Rodriguez Páez

Universidad Autónoma del Estado de México, México

clrodriguezp@uaemex.mx

(1) http://orcid.org/0000-0002-3856-0797

Yaroslaf Aarón Albarrán Fernández

Universidad Autónoma del Estado de México, México yaalbarranf@uaemex.mx

(D) http://orcid.org/0000-0002-2615-4740

Bernardo Soto Rivera

Hospital Regional $1^{\circ}$ de Octubre, México

bsotost@hotmail.com

(D) http://orcid.org/0000-0002-0994-3094

Alma Rebeca Gutiérrez Cruz

Hospital Regional $1^{\circ}$ de Octubre, México

rebekgut@hotmail.com

(1) http://orcid.org/0000-0003-4641-9424

Victor Manuel Duran López

Universidad Autónoma del Estado de México, México

vmduranl@uaemex.mx

(1) http://orcid.org/0000-0002-8457-5711

cienciaergosum.uaemex.mx 


\title{
RESUMEN
}

Se analiza la incidencia del tipo de cáncer y sus principales manifestaciones en la etapa terminal mediante la minería de datos. Se analizaron 288 datos de 22 pacientes, los cuales proporcionó la unidad de cuidados paliativos del Hospital Regional $1^{\circ}$ de Octubre. Las variables evaluadas fueron género, año, edad, diagnóstico y síntoma. Para la clasificación se utilizó el algoritmo J48 correspondiente al algoritmo C4.5. La mayor incidencia de mortalidad, de acuerdo con el tipo de cáncer, se da en el de pulmón, colon, recto, mama y el de próstata. En cuanto al dolor, se presenta en mayor medida en pacientes jóvenes, mientras que en pacientes de 65 años o menos 39\% y sólo en $23 \%$ de los restantes.

Palabras clave: minería de datos, cáncer, patrones, calidad de vida.

\begin{abstract}
The objective of this work is to analyze the incidence of the type of cancer and its main manifestations in the terminal stage through data mining. We analyzed 288 data from 22 patients, provided by the Palliative Care Unit of the Hospital Regional 1 de Octubre. The evaluated variables were gender, year, age, diagnosis and symptom. The algorithm J 48 corresponding to the algorithm C4.5 was used for the classification. The highest incidence of mortality according to the type of cancer is highlighted: lung, colon, rectum, breast and prostate. As for pain, it is higher in young patients, while in patients aged 65 or less, $39 \%$ and only $23 \%$ of the rest.
\end{abstract}

KEYWORDS: data mining, cancer, patterns, life quality.

\section{INTRODUCCIÓN}

La calidad de vida relacionada con la salud se define como la suma de factores físicos, emocionales y sociales que contribuyen al bienestar de una persona (Velarde-Jurado y Ávila-Figueroa, 2002; NIH, 2017). Un ámbito pionero y privilegiado en este tipo de estudios es la oncología, en donde se han diseñado instrumentos que buscan medirla. Fayers y Machin (2000) reportaron estudios en pacientes oncológicos debido al reconocimiento de que muchos cánceres avanzados son incurables (WHO, 1990), lo cual ha llevado a potenciar los cuidados paliativos (Guerrero, 2005) concebidos como el cuidado total activo proporcionado por un equipo multiprofesional y por familiares cuando por la enfermedad los pacientes ya no responden a tratamientos curativos (WHO, 1990, 2017; Marrone, 2002; Pessini y Bertachini, 2006; Esper Rueda, 2011). Sin embargo, no hay reportes de métricas que cumplan específicamente con los criterios psicométricos necesarios para cuidados paliativos, así como estadísticas reales sobre los pacientes que los requieren (Ascencio Huertas, 2015; Zamarrón Sanz et al., 2006). Por otra parte, Castro Aguilar et al. (2016) y Cuéllar Cisneros (2009) reportaron estudios de análisis estadístico y aplicaciones de minería de datos para obtener predicciones temporales basadas en agrupamientos que han permitido optimizar los recursos disponibles y priorizar el uso de diversos tratamientos para una misma patología. Asimismo, se han desarrollado modelos que han permitido obtener predicciones y brindar una explicación de los factores que conducen a estas patologías (Quesada et al., 2008). Por otra parte, Cabrera León et al. (2010) analizó un diseño muestral aleatorio como una técnica que aporta información sobre cuidados paliativos y es más precisa, y Rosete et al. (2009) analizaron los archivos y bitácoras para trabajar el nivel del conocimiento con el fin de descubrir patrones. Existen varias formas de cuantificar los síntomas (escala visual análoga y escala numérica), otras están diseñadas para síntomas y signos específicos (dolor: escala de Wong-Baker FACES; disnea: escala Borg CR10; 66 Manual de medicina paliativa 67 Depresión: HADS; confusión: método de evaluación CAM) y otras fueron creadas pensando en pacientes con compromiso de conciencia (ECAF: escala de confort de Edmonton). Algunas pueden ser aplicadas en forma general para varios síntomas y otras están diseñadas para síntomas y signos (López et al., 2009). Para apoyar el diseño de estas técnicas se ha incorporado el uso de las tecnologías de la información y comunicaciones (TIC) en los sistemas de salud, las cuales son consideradas como un facilitador para la transformación y mejoramiento en la calidad, mas nunca un fin en sí mismas; su incorporación en este sector ha permitido disponer de grandes bases de datos con información clínica, así como realizar tratamientos avanzados que incorporan cálculos estadísticos y análisis comparativos de series (Carnicero y Rojas, 2016). El aumento del volumen y variedad de información que se encuentra computarizada en bases de datos ha crecido en los últimos años; gran parte de esta información es 
histórica, pues representa transacciones o situaciones que se han producido, y puede ser de utilidad para comprender y unificar toda la información recuperada, proceso que generalmente se realiza a través de los almacenes de datos (White, 2001; Gutiérrez Cruz et al., 2016). La investigación y el desarrollo para analizar grandes volúmenes de datos se han hecho cada vez más necesarios, puesto que las ventajas aumentan cuando se cuenta con un volumen amplio y se descubre un conocimiento de este conjunto de datos (Monserrat y Chiotti, 2013; Riquelme et al., 2006).

La minería de datos se define como el procesamiento de los datos para encontrar patrones de comportamiento que sean de utilidad para la toma de decisiones; se relaciona de manera estrecha con la estadística al usar técnicas de muestreo y visualización de datos y depuración (Monserrat y Chiotti, 2013) y tener como materia prima las bases de datos (Molina Félix, 2014). El análisis de los datos puede proporcionar en conjunto un verdadero conocimiento que ayude en la toma de decisiones (Marcano Aular y Talavera Pereira, 2007).

En el alcance clínico la minería de datos resulta de la ayuda en la identificación y el diagnóstico de patologías para el descubrimiento de posibles correspondencias entre diversas enfermedades (Zamarrón Sanz et al., 2006; Hernández et al., 2004). Bajo este contexto, el Hospital Regional $1^{\circ}$ de Octubre (HR1O) desde hace poco más de ocho años ha implementado un programa de cuidados paliativo con prácticas enfocadas en controlar el dolor y atender los aspectos psicosociales del paciente y los familiares que lo acompañan en el proceso, es decir, evitar dolor y sufrimiento físico y emocional a pacientes con patologías en fase terminal, ya que cada año 10 millones de personas sufren de dolor por esta causa y 5.5 millones de ellos son por cáncer (Olden et al., 2011). El enfermo con cáncer terminal presenta alteraciones en otras facetas que deben ser consideradas; el manejo del dolor y otros síntomas representan sólo una parte de la ayuda que puede brindarse para mejorar la calidad de vida del paciente. Esta investigación tiene como objetivo analizar mediante la minería de datos la incidencia en tipos de cáncer y sus principales manifestaciones en pacientes terminales.

\section{Materiales y métodos}

Se analizaron 288 datos correspondiente a 22 expedientes clínicos de pacientes en el periodo 2009-2015 que proporcionó la Unidad de Cuidados Paliativos del Hospital Regional $1^{\circ}$ de Octubre de la Ciudad de México. La razón de tener esta cantidad de registros obedece a que en México no hay repositorios con base poblacional de cáncer que estén formalmente registrados; por lo regular se hacen de manera manual y en ocasiones se extravía la información (Reynoso y Mohar, 2014), aunado a que contados hospitales cuentan con una clínica del dolor y cuidados paliativos (García y Martínez, 2015).

La investigación se desarrolló bajo el enfoque cuantitativo de tipo descriptivo. Se utilizó el proceso general del KDD (Knowledge Discovery in Databases) para los datos recabados, de acuerdo con Marcano Aular y Talavera Pereira (2007), donde se contempla: entendimiento del problema, selección de datos y preprocesamiento y limpieza y transformación de datos. En todo el proceso de manejo de datos se les dio seguimiento constante y riguroso, que va desde el registro de ingreso a la unidad hasta los datos de fallecimiento de los pacientes.

\section{1. Entendimiento del problema}

En esta fase se requiere comprender con exactitud el problema al que se le va a dar solución por medio de la minería de datos. Esto permitirá recolectar la información necesaria para interpretar con asertividad los resultados encontrados (Gallardo, 2009).

\section{2. Selección y preprocesamiento de datos}

La selección y preprocesamiento de datos se obtuvo mediante la identificación de variables (género, año, edad, diagnóstico y síntomas) y la limpieza de datos consistió en seleccionar de manera coordinada los datos más relevantes 
del proceso y en el análisis de los expedientes clínicos; por el tipo de rigor, al recabar la información y procesarla, no se tuvieron inconsistencia de datos o valores atípicos, por lo que no fue necesario subsanar datos faltantes.

En la tabla 1 se muestra la descripción de cada uno de los atributos seleccionados como sexo, año, edad, diagnóstico y síntoma para el análisis y modelo de minería de datos.

\section{TABLA 1}

Descripción de atributos basados en el expediente clínico de los pacientes

\begin{tabular}{lll}
\hline Atributo & \multicolumn{1}{c}{ Definición } & \multicolumn{1}{c}{ Descripción } \\
\hline Dx & Diagnóstico & Se refiere al tipo de cáncer \\
Sexo & Sexo & Indica el género del paciente \\
Edad & Edad & Indica la edad del paciente \\
Fecha & Fecha & Fecha de ingreso del paciente \\
PromedioDolor & Promedio dolor & Indica la intensidad de dolor \\
ProDCF & Promedio de discapacidad & Indica cuando un paciente tiene una disminución de capacidades \\
& funcional & motrices \\
ProNauseas & Náuseas & Indica malestar a nivel intestinal \\
ProD & Dolor & Indica la intensidad de dolor \\
ProA & Ansiedad & Indica el nivel de angustia o ansiedad del paciente \\
ProS & Somnolencia & Indica el estado entre el sueño y la vigilia \\
ProFa & Fatiga & Indica el nivel de esfuerzo físico o mental del paciente \\
ProM & Malestar & Indica molestia o indisposición del paciente \\
ProFaire & Falta aire & Indica opresión y la falta de oxígeno del paciente \\
ProI & Insomnio & Indica el promedio en el trastorno del sueño del paciente \\
ProE & Estreñimiento & Indica el nivel de estreñimiento del paciente \\
ProAl & Alergia & Indica el nivel de alergia del paciente \\
ProH & Hiperventilación & Indica el nivel de hiperventilación del paciente \\
ProFi & Fiebre & Indica el nivel de fiebre del paciente \\
ProHS & Hipertensión & Indica el nivel de hipertensión del paciente \\
Flujonl & Flujo nasal & Indica el nivel de escurrimiento nasal del paciente \\
Flemaconsangre & Flema con sangre & Indica el nivel de flujo de flema con sangre del paciente \\
Cama & Cáncer de mama & Se refiere al cáncer de mama \\
Capu & Cáncer de pulmón & Se refiere al cáncer de pulmón \\
Capo & Cáncer de próstata & Se refiere al cáncer de próstata \\
Cacol & Cáncer de colon & Se refiere al cáncer de colon \\
Carec & Cáncer de recto & Se refiere al cáncer de recto \\
\hline & &
\end{tabular}

Fuente: elaboración propia.

\subsection{Análisis de datos}

Se realizó el análisis de datos de las variables evaluadas (género, edad y diagnóstico), así como para los tipos de cáncer mediante el software WEKA.[1]

\subsection{Clasificación}

Los datos incluidos en el conjunto en su mayoría eran categóricos, por lo que se optó por utilizar árboles de decisión; de los algoritmos disponibles, se utilizó el J48 correspondiente al algoritmo C4.5 (Sierra Araujo, 2006). 
En su ejecución se utilizaron especificaciones que por default tiene WEKA, así como el método de validación cruzada estratificada, también analizado por medio del algoritmo Apriori para los 288 datos y 19 instancias para obtener reglas coincidentes.

\section{Resultados Y Discusión}

De acuerdo con los resultados obtenidos en las diferentes pruebas realizadas en la etapa de minería de datos con las tareas de clasificación, el análisis de frecuencia de las variables estudiadas (edad, género y diagnóstico) para los 22 pacientes correspondía a $25.4 \%$ mujeres y $74.6 \%$ hombres (gráfica 1 a), con rango de edad de 39 a 55 años que equivale a $45.16 \%, 43.37 \%$ para edad de 56 a 71 y de 72 a 97 años $11.47 \%$ (gráfica 1 b); en lo referente a los tipos de cáncer: de próstata (CAPO), pulmón (CAPU), recto (CAREC), de mama (CAMA), y colon (CACOL), de los cuales su porcentaje de incidencia es 35, 33, 16.85, 10.04 y 3.94, respectivamente (figura 1c). Lo anterior promueve el descubrimiento de patrones inesperados que conllevan al surgimiento de la etapa de minería de datos, en donde pueden ser aplicados algoritmos de a) clasificación (Witten et al., 2000), b) clustering, c) patrones secuenciales $y d$ ) asociaciones. Dentro de las variables analizadas, el género se considera con una importante tasa de mortalidad en determinadas enfermedades (Rodríguez Jiménez et al., 2009; Borràs, 2015), así como la edad debido a su incidencia en enfermedades crónicas si se toma en cuenta que los pacientes con edad avanzada cursan con menor expectativa de supervivencia, aun en etapas tempranas, cuando se comparan con los de menor edad (González et al., 2002; Colson et al., 2005; OMS, 2017).

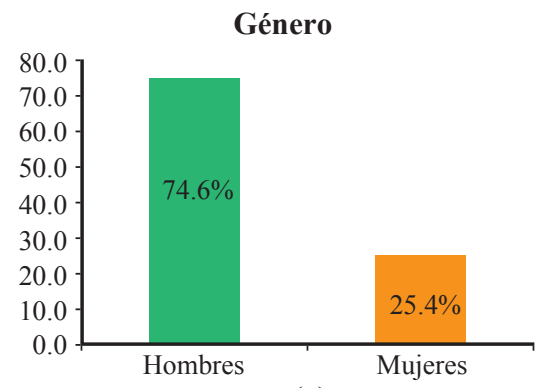

(a)
Edad

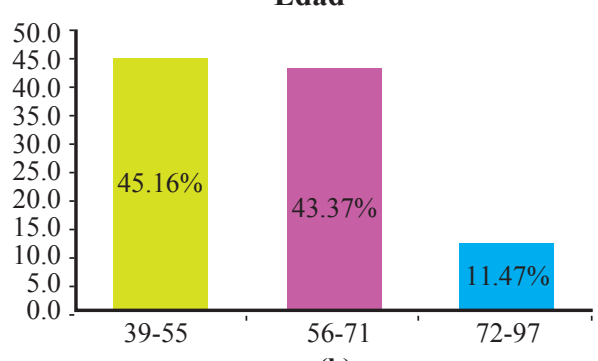

(b)

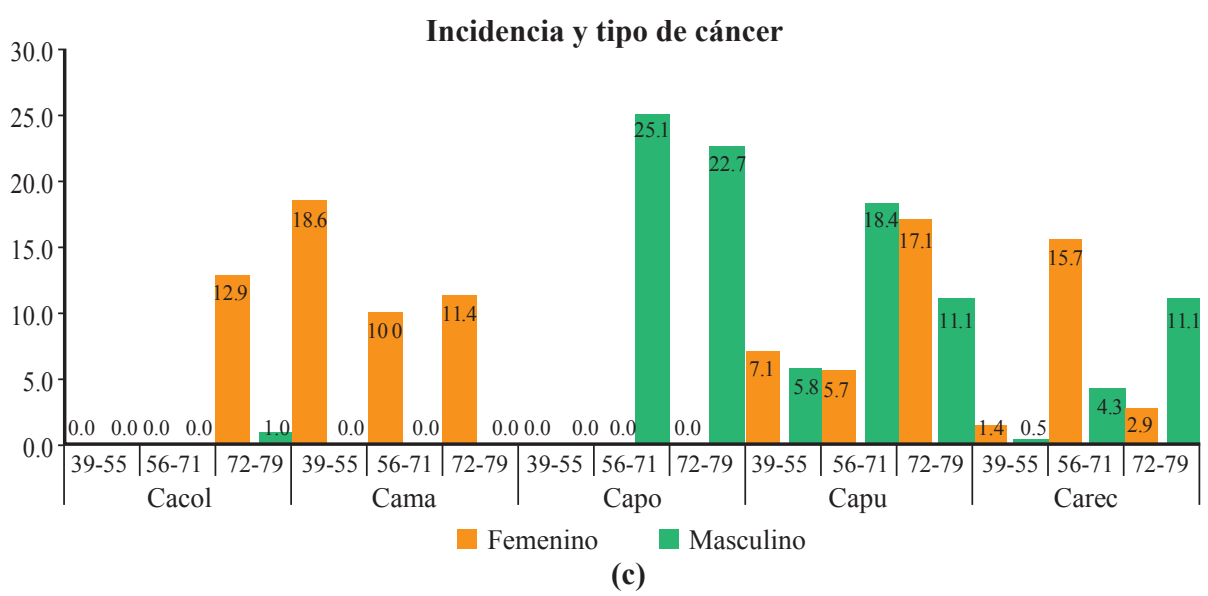

GRÁFICA 1

Resultados de las diferentes pruebas realizadas

Fuente: elaboración propia.

Nota: a) población de estudio por género, $b$ ) rangos de edad de la población afectada, $c$ ) incidencia y tipos de cáncer por género y rango de edad. 
En la tabla 2 se muestran las reglas obtenidas con el algoritmo Apriori aplicado para identificar los síntomas con mayor incidencia en los distintitos tipos de cáncer.

TABLA 2

Algoritmo Apriori aplicado para identificar los síntomas de mayor incidencia en los distintos tipos de cáncer 1. ProHS=HSlevemoderado $286==>$ Flemaconsangre=FSlevemoderado $286<$ conf:(1)> lift:(1.18) lev:(0.13) [43] conv:(43.03)

2. Flujonl=FLlevemoderado $283==>$ Flemaconsangre=FSlevemoderado $283<$ conf:(1) $>$ lift:(1.18) lev:(0.13) [42] conv:(42.58)

3. ProFi=FIlevemoderado $281==>$ ProHS=HSlevemoderado $281<$ conf:(1)> lift:(1.19) lev:(0.13) [43] conv:(43.93)

4. ProFi=FIlevemoderado $281==>$ Flemaconsangre=FSlevemoderado $281<$ conf:(1)> lift:(1.18) lev:(0.12) [42] conv:(42.27)

5. ProFi=FIlevemoderado Flemaconsangre $=$ FSlevemoderado $281==>$ ProHS=HSlevemoderado $281<$ conf:(1)> lift:(1.19) lev:(0.13) [43] conv:(43.93)

Fuente: elaboración propia.

En las reglas obtenidas por el algoritmo Apriori se presenta combinación entre fiebre, flujo nasal, hipertensión y flema con sangre; todas las reglas mencionadas mantienen una confiabilidad de 1 , la cual indica que el resultado es muy confiable. En una prueba focalizada (usando el algoritmo J48) que contempla los padecimientos señalados se mostró que el cáncer de próstata, de pulmón y de mama los involucraban en su mayoría; lo anterior coincide con los resultados obtenidos por Bruera (2008) y Coyle et al. (1990). Referente a la intensidad de dolor por tipo de cáncer, se observaron los resultados mediante el algoritmo REPTree (figura 1), en donde ProDCF indica que hay una intensa disminución en la capacidad funcional, la cual se denota con mayor incidencia en el cáncer de mama y de pulmón para ejecutar acciones o tareas, y las restricciones son un problema para participar en situaciones vitales.

Entre los distintos tipos de cáncer reportados en la unidad se tiene que la mayor incidencia de mortalidad se presenta en pulmón, colon, recto, mama y próstata, lo cual coincide con Fonseca et al., 2013; NIH, 2017, en tanto que los síntomas predominantes entre ellos resaltan por ser los más comunes en la etapa final de cáncer de pulmón, como lo menciona ACS (2016). El cáncer de colon presenta alteraciones generales como anemia, fatiga o cansancio y falta de apetito.

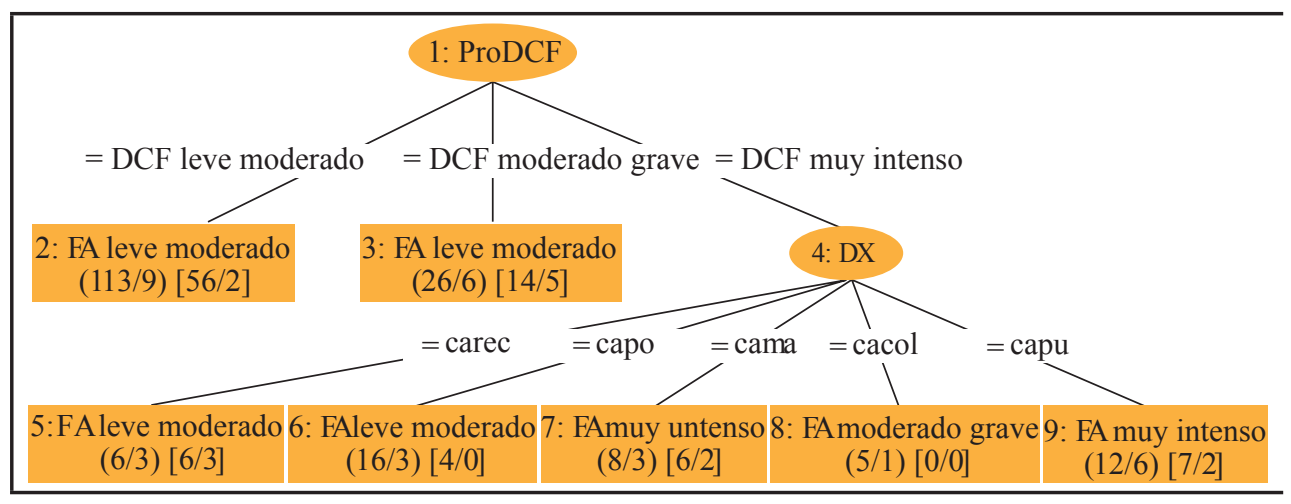

FIGURA 1

Resultados obtenidos con base en el tipo de diagnóstico y la intensidad de dolor de cada uno clasificado con el algoritmo REPTree

Fuente: elaboración propia.

En lo referente al cáncer de mama femenino también prevalece el cansancio o el dolor óseo localizado; para el cáncer de próstata se reporta anemia, además de hinchazón en los miembros inferiores e insuficiencia renal que 
suelen aparecer en los estadios avanzados de la enfermedad (AECC, 2017). El dolor es una constante que afecta entre $70 \%$ a $90 \%$ de los pacientes en fase terminal con cáncer, por lo que su manejo inadecuado es un grave problema de salud pública (Astudillo Alarcón et al., 2009); los pacientes con cuidados paliativos requieren un modelo de intervención que comprenda medidas farmacológicas, psicoterapéuticas y rehabilitadoras orientado a disminuir el dolor (IASP, 2015); los tipos de cáncer aquí reportados con mayor intensidad coinciden con MSC (2005). Aunque el dolor es clínicamente significativo, aumenta cuando se acerca la muerte; la prevalencia del dolor disminuye conforme incrementa la edad. Se resalta que la presencia del dolor en los pacientes dos años antes de la muerte es mayor en los jóvenes, seguido por los de 65 años o menos y por último con los de 86 años o más. En el caso de los adultos mayores Smith et al. (2010) y Castanera et al. (2019) mencionan que las herramientas empleadas en la valoración de dolor y otros síntomas no son apropiadas para este último grupo. En relación con el género, la opinión es bastante igualitaria entre quienes valoran la situación de los cuidados paliativos como "buena" o "muy buena", mientras que la valoración "mala" o "muy mala" es ligeramente más alta entre los hombres. La única diferencia que es estadísticamente significativa es la valoración de las mujeres como "muy buena” por encima de la media, mientras que significativamente por debajo se sitúan los hombres que la califican en esta categoría (Cabrera León et al., 2010).

\section{Conclusiones}

Con base a los resultados obtenidos de los 22 pacientes se observa que los tipos de cáncer con mayor incidencia de mortalidad son el de pulmón, colon, recto, mama y próstata, los cuales son reportados por la Sociedad Americana Contra el Cáncer y el Instituto Nacional del Cáncer como los más comunes. Los pacientes con edad avanzada son quienes presentan menor expectativa de supervivencia. La intensidad de dolor por tipo se denota con mayor incidencia en el de mama y de pulmón afectando así la calidad de vida del paciente. Con esta evidencia resalta la necesidad de seguir analizando distintos tipos de datos con las técnicas de minería de datos. En este sentido, se logra concluir que esta técnica es una de las más recientes y prometedoras de investigación para el análisis de datos en el área de la salud, y en general en otras áreas.

Entre los principales síntomas manifestados en la etapa terminal se encuentra una combinación entre fiebre, flujo nasal, hipertensión y flema con sangre, que muestra la necesidad del tratamiento paliativo para este tipo de pacientes.

Cabe resaltar que en México falta darle a los profesionales de la salud la importancia en su formación y capacitación en cuidados paliativos y su aplicación en los tres niveles de salud que lo requieren.

\section{AnÁlisis PROSPeCtivo}

Los resultados de este artículo proporcionan elementos de interés para discutir sobre el valor de identificar o descubrir relaciones en información aparentemente "oculta" con el objetivo de proponer estrategias para contrarrestar problemas de salud o prevenir futuras complicaciones y contribuir así a la mejora en la calidad de vida de la población, como sería el caso de la minería de datos en el área de la salud, que ha demostrado aplicabilidad en la detección precoz y prevención de enfermedades, para el análisis de marcadores genéticos, para conocer la probabilidad de una respuesta satisfactoria a un tratamiento médico (Marchán et al., 2011), determinar factores influyentes en el padecimiento de diabetes (Rosete Suárez et al., 2009) e hipertensión (Dávila Hernández y Sánchez Corales, 2012), así como en las relaciones entre determinadas variables socioeconómicas y las cuatro causas principales de muerte por cáncer (colorrectal, pulmón, mama y próstata). En los Estados Unidos permitió conocer qué áreas de salud con índices de educación bajo, alto desempleo y trabajos mal pagados tenían las mayores tasas de mortalidad por esta enfermedad (Zhao et al., 2011) y evidenciar la presencia de diagnósticos asociados al cáncer, para lo cual se agrupó a la población por edad, grupo etario, régimen de salud, género, zona de residencia, estrato, etnia, estado civil; resalta que los tipos de tumores malignos en hombres eran de estómago, 
colon y la laringe, mientras que entre las mujeres fueron de colon, estómago y cerebro. En la administración de fármacos en pacientes con síntomas de enfermedad cardiovascular se usaron las variables de presión arterial, índice de colesterol, azúcar en la sangre, alergias a antibióticos y otras alergias (Solarte Martínez y Soto Mejía, 2011). Por lo anterior, es importante reconocer que todos estos trabajos van encaminados a obtener conocimiento de los datos. Este conocimiento puede tener un valor científico o investigador que ayude a determinar causas de determinadas patologías o a identificar poblaciones de riesgo y así, por un lado, contribuir en la detección precoz de enfermedades y, por otro lado, en la gestión de sistemas de salud y también de gestión hospitalaria es una herramienta útil para la toma de decisiones y la optimización de recursos.

\section{REFERENCIAS}

Ascencio Huertas, L. (2015). Adaptación en español de la escala de actitudes ante cuidados paliativos: confiabilidad y análisis factorial. Psicooncología, 12(2-3), 367-381.

AECC (Asociación Española Contra el Cáncer). (2017). Sintomas del cáncer. Disponible en www.aecc.es

ACS (American Cancer Society). (2016). Signos y síntomas del cáncer de pulmón microcítico. Disponible en https://www.cancer.org/content/dam/CRC/PDF/Public/9002.00.pdf

Astudillo Alarcón, W., Díaz-Albo, E., García Calleja, J. M., Mendinueta, C., Granja, P., Fuente Hontañón, C. de la Orbegozo, A., Urdaneta, E., Salinas-Martín, A., Montiano, E., González Escalada, J. R. y Torres, L. M. (2009). Cuidados paliativos y tratamiento del dolor en la solidaridad internacional: international solidarity. Revista de la Sociedad Española del Dolor, 16(4), 246-255.

Borràs, J. M. (2015). La perspectiva del género en el cáncer: una visión relevante necesaria. Arbor, 1, 91-773.

Bruera, E. (2008). Symptom control in patients with cancer. Journal of Psychosocial Oncology, 8(2-3), $47-73$

Cabrera León, A., Cía Ramos, R., Escudero Carretero, M. J., Fernández López, A., García Toyos, N., Ruiz Román, P., Sanz Amores, R. (2010). Cuidados paliativos en Andalucía: valoración de profesionales del SSPA. Sevilla: Consejería de salud.

Castanera Duro, Aaron; Díaz, Verónica ; Cívico, Ana ; Pujolras, María ; Lamigueiro, Andrea \& Guadalupe, María \& Cuesta, García \& Pumarola, Concepción. (2019). PERCEPCIÓN DEL DOLOR EN PACIENTES POSTOPERADOS DE CIRUGÍA CARDIACA. 60. 22-26.

Carnicero, J. y Rojas, D. (2016). La explotación de datos de salud: retos, oportunidades y límites. Pamplona: Sociedad Española de Informática de la Salud. Disponible en http://www.seis.es.

Castro Aguilar, G. F., Pérez Pupo, I., Piñero Pérez, P., Martínez, N. y Cruz Castillo, Y. (2016). Aplicación de la minería de datos anómalos en organizaciones orientadas a proyectos. Revista Cubana de Ciencias Informáticas, 10(1), 195-209.

Colson, B., Marcotte, P. \& Savard, G. . (2005). Bilevel Programming. A survey. 4OR 3, 87-107

Coyle, N., Adelhart, J., \& Foley, K. (1990). Character of terminal illness in the advanced cancer patient. Pain and other symptoms during the last four weeks of life.Journal of Pain and Symptom Management, 5(2), 83-89.

Cuéllar Cisneros, L. (2009). Intervención en tribuna de la senadora Lorena Cuéllar Cisneros del grupo parlamentario del PRD. Presentación de iniciativa con modificación a la ley general de salud en materia de prevención del dolor en pacientes terminales a través de cuidados paliativos. Disponible en https://www.senado. gob.mx/64/intervenciones/595/por_periodo/LXII/3/Primer_Periodo_Ordinario

Dávila Hernández, F. y Sánchez Corales, Y. (2012). Técnicas de minería de datos aplicadas al diagnóstico de entidades clínicas. Revista Cubana de Informática Médica, 4(2), 174-183. Disponible http://scielo.sld.cu/ 
scielo.php?script=sci_arttext\&pid=S1684-18592012000200007\&lng=es\&tlng=es.

Esper Rueda, J. A. (2011). Cuidados paliativos en pacientes con cáncer: una necesidad actual y vigente. Médicas UIS, 24(1), 9-16.

Fayers, M. P., \& Machin, D. (2000). Quality of Life: The Assessment, Analysis and Interpretation of Patient-reported Outcomes. John Unily \& Sons Ltd.

Fonseca, M. C., Schlack C. V., Mera, E. M., Muñoz, S. O., \& Peña J. L. (2013). Assessment of quality of life in patients with terminal cancer. Revista Chilena de Cirugía, 65(4), 321-328.

Gallardo,J. (2009).Metodología para el desarrollo deproyectos en minería de datos CRISP-DM.Disponible enhttp:// www.oldemarrodriguez.com/yahoo_site_admin/assets/docs/Documento_CRISP-DM.2385037

García, P. y Martínez, P. (2015). Experiencia en el primer año de implementación de una clínica del dolor y cuidados paliativos en un Hospital General Regional. Revista Mexicana de Anestesiología, 38(1), 15-20.

González, J. R., Llorca, F. J. y Moreno, V. (2002). Algunos aspectos metodológicos sobre los modelos edad-período-cohorte: aplicación a las tendencias de mortalidad por cáncer. Gaceta Sanitaria, 16(3), 267-273.

Guerrero, M. (2005). La paliación en los albores del nuevo siglo. Tema monográfico: Cuidados paliativos en el enfermo terminal. ARS Médica, 11, 11-3.

Gutiérrez Cruz, D., Rico Molina, R., Rodríguez Páez, L., Vilchis Hernández, K., Soto Rivera, B., Albarrán Fernández, Y., Gutiérrez Cruz, A. y Ortega, M. (2016). Oncological analysis using data mining. Research in Computing Science, 121, 139-149.

Hernández, J., Ramírez, M. J. y Ferri, C. (2004). Introducción a la minería de datos. Madrid: Pearson-Prentice Hall.

IASP (International Association for the Study of Pain). (2015). Define el dolor como "una sensación o experiencia desagradable, sensorial y emocional que se asocia a una lesión tisular verdadera o potencial". Disponible en https://www.iasp-pain.org/.

López, R., Nervi, F. y Taboada, P. (2009). Manual de medicina paliativa. Santiago. Disponible en http://148.202.167.116:8080/jspui/bitstream/123456789/935/3/Manual_Medicina_Paliativa_PUC.pdf

Marcano Aular, Y. J. y Talavera Pereira, R. (2007). Minería de datos como soporte a la toma de decisiones empresariales. Opción, 23(52), 104-118.

Marchán, E., Salcedo, J., Aza, T., Figuera, L., Martínez de Pisón, F. y Guillén, P. (2011). Reglas de asociación para determinar factores de riesgo epidemiológico de transmisión de la enfermedad de Chagas. Ciencia e Ingeniería.

Marrone, R. (2002). Spiritual issues and quality of life assessment in cancer care. Death Studies, 26, 743-56.

Molina Félix, L. C. (2014). Data Mining: torturando a los datos hasta que confiesen. Disponible en http:// www.uoc.edu/web/esp/art/uoc/molina1102/molina1 102.html

Monserrat, S. y Chiotti, O. (2013). Mineria de datos en base de datos de servicios de salud. Disponible en http:// conaiisi.unsl.edu.ar/2013/132-505-1-DR.pdf

NIH. (2017). Tipos comunes de cáncer. Instituto Nacional del Cáncer. https://www.cancer.gov/espanol/ tipos/comunes

Olden, A. M., Holloway, R., Ladwig, S., Quill, T. E., \& van Wijngaarden, E. (2011). Palliative care needs and symptom patterns of hospitalized elders referred for consultation. Journal of Pain and Symptom Management, 42(3), 410-418.

Pessini, L. y Bertachini, L. (2006). Nuevas perspectivas en cuidados paliativos. Acta Bioethica, 2(12), 231-242.

Quesada, A. Y., Wong Pérez, D. y Rosete Suárez, A. (2008). Minería de datos aplicada a la gestión hospitalaria. 14 Convención Científica de Ingeniería y Arquitectura. CUJAE. 
Reynoso, N. y Mohar, A. (2014). El cáncer en México: propuestas para su control. Salud pública de México, 56(5).

Riquelme, J. C., Ruiz, R. y Gilbert, K. (2006). Conceptos y tendencias inteligencia artificial. Revista Iberoamericana de Inteligencia Artificial, 10(29), 11-18.

Rodríguez Jiménez, P., Fernández Alfonso, J., Delgado Pérez, L., Garrote Rodríguez, I., Morales Rigau, J. M. y Achiong Estupiñán, F. J. (2009). Mortalidad por cáncer y condición de género. Centro Provincial de Higiene y Epidemiología. Matanzas, 31(1), 1-10.

Rosete Suárez, A., Rodríguez Díaz, A. y Acosta Sánchez, R. (2009). Predicción de pacientes diabéticos. Preprocesado para minería de datos. Revista Cubana de Informática Médica, 9(1). Disponible en http:// www.rcim.sld.cu/revista_18/articulos_htm/prediccionpaciente.htm\#t

Sierra Araujo, B. (2006). Aprendizaje automático: conceptos básicos y avanzados. Aspectos prácticos utilizando el software Weka. Prentice-Hall.

Smith, A. K., Cenzer, I. S., Knight, S. J., Puntillo, K. A., Widera, E., Williams, B. A., ... Covinsky, K. E. (2010). The epidemiology of pain during the last two years of life. Annals of Internal Medicine, 153(9), 563-569.

Solarte Martínez, G. y Soto Mejía, J. (2011). Arboles de decisiones en el diagnóstico de enfermedades cardiovasculares. Scientia et Technica, 16(49), 104-109.

Velarde-Jurado, E. y Ávila-Figueroa, C. (2002). Evaluacion de la calidad de vida. Salud Pública de México, 44(4), 349-361.

White, C. J. (2001). IBM Enterprise Analytics for the Intelligent E-Business. USA IBM Press.

WHO (World Health Organization). (1990). Cancer pain relief and palliative care: report of a WHO expert committee [meeting held in Geneva from 3 to 10 July 1989]. Geneva: World Health Organization. Disponible en http://www.who.int/iris/handle/10665/39524

WHO (World Health Organization) (2017). Cuidadospaliativos. Disponible en https://www.who.int/es/news$\mathrm{room} /$ fact-sheets/detail/palliative-care.

Witten, I., Frank, E., \& Hall, M. (2000). Data mining: Practical machine learning tools and techniques. Morgan Kaufman Publishers.

Zamarrón Sanz, C., García Paz, V., Calvo Álvarez, U., Pichel Guerrero, F. y Rodríguez Suárez, J. R. (2006). Aplicación de la minería de datos al estudio de las alteraciones respiratorias durante el sueño. Revista Pneuma, 6, 156-166.

Zhao, F., Zhu, R., Zhang, L. J., Zhang, Z. J., Li, Y. P., He, M. Z., Zhou, Y, B., Guo, J. G., Zhao, G. M., \& Jiang, Q. W. (2011). Application of satscan in detection of schistosomiasis clusters in marshland and lake areas. Chinese Journal of Schistosomiasis Control, 23, 28-31. in Chinese. Retrieved from https://www.ncbi.nlm. nih.gov/pubmed/22164371

\section{Notas}

[1] Disponible en http://www.cs.waikato.ac.nz/ml/weka/

\section{BY-NC-ND}

